patients with a diagnosis of AIH who required the addition of tacrolimus as a third line agent.

Methods The tacrolimus database for the Regional Liver Unit, Royal Victoria Hospital was reviewed to identify all patients with AIH who had been treated with tacrolimus from Jan 2010 until August 2017. Records were cross referenced with the diagnostic coding department. Demographic details, indications for tacrolimus therapy, clinical and biochemical outcomes were recorded.

Results 30 patients were identified (24 (80\%) female, mean age 40.7 years, range $19-81$ years). 27 of the 30 patients were initially treated with azathioprine of whom 21 (78\%) discontinued treatment due to adverse effects including blood dyscrasias and $6(22 \%)$ were switched to tacrolimus due treatment failure. Three of 30 patients were started on tacrolimus instead of azathioprine or mycophenolate. Two of these patients had previous episodes of pancytopenia at the time of commencing treatment for $\mathrm{AIH}$ and azathioprine/mycophenolate were excluded as a treatment option. One of the patients was commenced on prednisolone and tacrolimus without another steroid sparing agent trailed for other reasons. 26 $(87 \%)$ of 30 patients remain on tacrolimus, of whom 11 $(42 \%)$ had normalisation of transaminases and a further 12 $(46 \%)$ had improvement of transaminases. Liver function tests in the $3(11.5 \%)$ remaining patients were deranged but static. Of note all three had established cirrhosis at the time of AIH diagnosis. Of the four whose tacrolimus therapy was discontinued, two stopped due to side effects, 1 is deceased (not tacrolimus related) and one stopped due to commencing infliximab for IBD.

Conclusions Tacrolimus is a safe and well tolerated treatment for AIH when first line therapy has failed. In the cohort observed, only $6 \%$ failed to tolerate tacrolimus and biochemical parameters were improved or normalised in $88 \%$ of patients who remained on tacrolimus therapy.

\section{PTH-085 USING ELF TESTS IN PRIMARY AND SECONDARY CARE TO IDENTIFY PATIENTS WITH ADVANCE FIBROSIS}

Charlotte Cook*, Alison Burridge, Janisha Patel. University Hospital Southampton, Southampton, UK

\subsection{6/gutjpl-2018-BSGAbstracts.241}

Introduction In 2016 NICE NAFLD guidelines recommended Enhance Liver Fibrosis (ELF) as a validated test to assess advanced liver fibrosis.

This study aims

ELF tests were performed, 166 from by GP practices and 175 by UHS hepatology service. 89 were from other organisations. to assess the relationship with standard investigations used in a hepatology clinic for NAFLD patients (including transient elastography) and the newly commissioned ELF test in a real world setting.

Methods The study looked at all new patients diagnosed with NAFLD via the hepatology outpatient clinic at University Hospital Southampton between November 2016 and May 2017.

Each patient had their demographics (age, gender, weight) and comorbidities (diabetes, hypertension, dyslipidaemia) assessed. Transient elastography, USS and ELF Result (when performed) were reviewed.

The ELF was categorised as no/mild fibrosis if $<7.5$, moderate fibrosis if $\geq 7.5$ and $\leq 10.5$, advanced fibrosis if $\geq 10.51$.
The ELF tests requested over the same period were analysed review the source of the request.

Results 175 people were diagnosed with NAFLD through hepatology clinic. All patients had transient elastography performed. The mean LSM Result was 14.6 (range 3.3-75), with a mean CAP score of 313.6 (range 100-400). Of 175 new diagnoses, 101 patients were male (mean weight $99 \mathrm{~kg}$, median age 52 years) and 74 were female $(87.1 \mathrm{~kg}$ and 57 years).

69 patients had type 2 diabetes mellitus, 74 had hypertension and 66 had dyslipidaemia. 30 patients had all 3 (16 were female with a mean weight $96.5 \mathrm{~kg}$ and mean LSM $18.5 \mathrm{kPa}$ and 14 were male, $100.7 \mathrm{~kg}$ and $19.3 \mathrm{kPa}$ ).

With transient elastography, 59 patients had LSM $<6 \mathrm{kPa}$, 68 were $6.1-12 \mathrm{kPa}, 23$ were $12.1-20 \mathrm{kPa}$ and $25>20.1 \mathrm{kPa}$. This suggested $48(27 \%)$ patients had advanced fibrosis or cirrhosis. The remaining 127 (73\%) patients did not require hepatology review.

430The GP cohort had 18 (11\%) patients with an ELF test $\geq 10.5$ requiring hepatology review. 36 (21\%) patients seen in the hepatology clinic had an ELF $>10.51$, the remaining 139 (79\%) patient did not require a hepatology review.

Conclusions This study looked at the cohort of new diagnoses of NAFLD in a teaching hospital using standard tests and ELF score. Currently, access to transient elastography is secondary care based. The preliminary data in this study shows that the ELF test is a good first line investigation for GPs suspecting NAFLD in a patient with type 2 diabetes and obesity or incidental finding of fatty liver. It promotes the need to look beyond the routine liver panel test and identify the aetiology of liver disease and assess extent of liver fibrosis; in turn, to generate appropriate secondary care referrals and incorporate efficiency. Further assessment of the use of ELF in this setting continues

\section{PTH-086 VIRTUAL HEPATITIS B CLINICS SIGNIFICANTLY IMPROVE COST AND CLINICAL EFFECTIVENESS}

Douglas Corrigall* Sarah Fairclough, Linda Porter, Katrina White, Denise Killworth, Gavin Wright. Basildon and Thurrock University Hospital, Basildon, UK

10.1136/gutjnl-2018-BSGAbstracts.242

Introduction Chronic Hepatitis B (HBV) infection requires regular hepatology review to assess for potential flares, need for therapy and/or progression to advanced liver disease and its complications. The majority of patients are chronic carriers who feel perfectly well and can find repeated clinic visits difficult, unnecessary and impinge on other commitments (e.g. employment, social and child care). As a Result there is a high rate of missed appointments and or patients lost to follow up, with the potential for missed viral flares. A nurse-led virtual clinic allows remote monitoring of these patients as recommended by national and international guidelines. ${ }^{1} 2$

Methods Patients with stable chronic HBV suitable for telephone clinic follow-up were identified. The criteria used were: not on/received HBV therapy, no significant comorbidities or risk factors for liver disease, low viral load $(<2000 \mathrm{iu} / \mathrm{ml}), \mathrm{F} 0 /$ 1 disease, low and stable alpha-fetoprotein (AFP), unremarkable imaging, and no transaminitis/native liver derangement.

Over a 2.5 year period from 01.02.2014, patients meeting the criteria were offered 6-monthly virtual clinic review. Patients had surveillance blood test within 4 weeks of their 
apportioned telephone review to discuss Results and/or symptoms. As per NICE guidance annual Fibroscan was organised through a dedicated clinic. If any clinical and/or biochemical concerns arose a face-to-face review in clinic is arranged.

Results Over this period 45 patients received 6-monthly virtual HBV clinic review. Bloods showed a median peak ALT $36 \mathrm{u} / \mathrm{L}$ $(0-41 \mathrm{u} / \mathrm{L})$, median viral load $62 \mathrm{iu} / \mathrm{ml}$. and median AFP 2.7. Only $3 / 45$ required face-to-face follow up due to a possible flare, with $2 / 45$ eventually requiring an antiviral agent (Tenofovir). No patients required admission and/or access to other acute and/or community services due to their liver disease during this period. The cost savings were considerable. 6 monthly reviews in a face-to-face clinic over the 2.5 year period would have cost an estimated $£ 27636$. The cost of running a virtual telephone clinic for the same period was $£ 5,517$; a saving of $501 \%$. There were only 5 missed blood tests ahead of the clinic appointment. This compares to the $22 \%$ DNA rate in our general hepatology clinics.

Conclusions A nurse-led virtual clinic provides high-quality, cost-effective care to patients and improves HBV monitoring. Better compliance allows for detection of flares which could require antiviral treatment. With the prospect of new treatments on the horizon it is important these patients remain under the care of a hepatology team and a virtual clinic model allows them to remain engaged with Liver services.

\section{PTH-087 IMPROVING HEPATITIS C DETECTION AND TREATMENT VIA COMMUNITY-BASED SERVICES}

Douglas Corrigall*, Linda Porter, Sarah Fairclough, Katrina White, Denise Killworth, Gavin Wright. Basildon And Thurrock University Hospital, Basildon, UK

\subsection{6/gutjnl-2018-BSGAbstracts.243}

Introduction With the advent of highly efficacious Direct Acting Antiviral (DAA) Hepatitis C (HCV) therapy, the World Health Organisation project global HCV eradication by 2030 and NHS-England is targeting HCV eradication nationally by 2025. Despite a nationwide HCV operational delivery network it is estimated that $\sim 90 \%$ of those infected in England are not actively engaged with secondary/tertiary HCV services providing therapy and many, often asymptomatic, remain untested and undiagnosed. These patients do engage with primary care; but with increasing pressures on these services and absence of national screening, detection and onward referral to treatment centres are poor. This is a barrier to achieving this target, and the new frontier for HCV services is improved detection and engagement in the community. People with a history of intravenous drug and alcohol misuse have a high prevalence of $\mathrm{HCV}$, are often sexually active with higher rates of transmission, have limited access to and/or engagement with HCV services, but often do attend community drug and alcohol services (CDAS). Our aims were to increase identification and treatment of patients with HCV by engaging these individuals within a community-based setting.

Methods Over one year (August 2015-2016), in partnership with five local CDAS we provided onsite nurse-led consultation, counselling, screening and risk stratification through noninvasive measurement of liver stiffness (fibroscan), dried blood spot screening (HBV/HCV/HIV serology, HCV RNA, T-spot), and referral to secondary care for initiation of approved DAA therapy and ongoing management of any concomitant chronic liver disease.
Results 174 CDAS service-users were screened and 123 (70\%) were diagnosed as HCV RNA positive; 54\% Genotype 3\% and $46 \%$ Genotype 1. Median fibroscan score 7.1 Kpa, with $21(12 \%)$ had a fibroscan Result suggestive of cirrhosis and were prioritised to treatment according to National guidance via our NHS-England HCV ODN.

To-date $86(70 \%)$ pf the HCV positive patients have attended our clinic for consideration of access to DAA therapy.

Conclusions This community-based pilot had a significant rate of detection (70\%), and excellent conversion to secondary care clinic review (70\%). However, the majority of our patients had low levels of fibrosis and as NHS England policy over that period prioritised for patients with advanced disease, this cohort did not receive immediate access to treatment from the ODN over the time of the project, with Resultant disengagement by many from secondary care. Given recent changes in treatment access prioritisation we are now actively reengaging this group which represents an ongoing challenge.

\section{PTH-088 'MORE IS LESS' - PRESENTING WITH ACUTE VARICEAL BLEEDING}

Benjamin Crooks*, Helen White, Vivek Goodoory, Thomas Butler, Mark Murgatroyd, Javaid Iqbal. Wythenshawe Hospital, Manchester University Foundation Trust, Manchester, UK

\subsection{6/gutjnl-2018-BSGAbstracts.244}

Introduction Acute variceal bleeding (AVB) has historically accounted for up to $10 \%$ of all GI bleeds necessitating emergency out of hours endoscopy. These patients have a significantly poorer prognosis and higher re-bleed rate than nonvariceal GI haemorrhage. In the last 3 years we have anecdotally noticed a significant reduction in AVB necessitating emergency endoscopy. This has coincided with the employment of dedicated hepatologists. We sought to qualify and quantify this reduction with the employment of one and then a second hepatologist at a university teaching hospital 18 months apart. Methods This was a retrospective review identifying all AVB patients who underwent emergency endoscopy over a 3 year period between January 2015 and December 2017. Data was collected from the electronic database and the GI reporting tool. This included endoscopic findings, therapy performed and whether there was a previous history of AVB requiring endoscopy. A dedicated hepatologist was employed in January 2015 (period 1; 18 months) and a second hepatologist June 2016 (period 2; 18 months).

Results Prior to a dedicated hepatologist all patients were followed up by general gastroenterologists. There was no dedicated variceal banding programme. There were up to 300 acute GI bleed endoscopies a year with approximately $10 \%$ due to AVB. Both hepatologists began performing weekly dedicated oesophageal variceal screening and treatment endoscopy lists (between 1-2/week). During period 1, there were $30 \mathrm{AVB} ; 27 / 30$ (90\%) received therapy, in the remaining 3, banding could not be applied due to poor views and injection therapy or sengstaken tube placement was performed. Of those presenting; 21/30 (70\%) had previous OGD and banding but only $8 / 21(38 \%)$ had previously been on banding program.

During period 2, there were 20 AVB; $19 / 20$ (95\%) received therapy. $12 / 20(60 \%)$ had previous OGD and banding, and only $3 / 20(15 \%)$ were on a dedicated banding programme. 